\title{
The Prevalence of Metabolic Syndrome In Non-alcoholic Fatty Liver Disease; A Population-Based Study
}

\author{
Mohammad Reza Fattahi ${ }^{1}$, Ramin Niknam ${ }^{1 *}$, Alireza Safarpour ${ }^{1}$, Masood Sepehrimanesh ${ }^{1}$, \\ Mehrzad Lotfi ${ }^{2}$
}

1. Gastroenterohepatology Research Center, Shiraz University of Medical Sciences, Shiraz, Iran

2. Medical Imaging Research Center, Shiraz University of Medical Sciences, Shiraz, Iran

\footnotetext{
* Corresponding Author:

Ramin Niknam, MD

Assistant professor of Gastroenterology and Hepatology, Gastroenterohepathology Research Center, Shiraz University of Medical Sciences, P.O. Box: 71935-1311, Shiraz, Iran

Telefax:+98 7136474263

Email: niknamramin@yahoo.com

Received: 18 Nov. 2015

Accepted: 10 Feb. 2016
}

\section{ABSTRACT}

\section{BACKGROUND}

Some evidence, not in large study populations, suggests that nonalcoholic fatty liver disease (NAFLD) and metabolic syndrome (MetS) share common interactions. We aimed to determine the prevalence of NAFLD and MetS in a large population registered to Kavar Cohort Study center. We also assessed the role of each component of MetS in NAFLD existence.

\section{METHODS}

Data were obtained from 3415 volunteers who called and refereed to our center. Complete anthropometric and laboratory measurement and abdominal ultrasonography was done for these individuals to screen NAFLD and its grade. A questionnaire was also used to obtain information on demographical and medical history and alcohol consumption. MetS was defined in all participants based on the National Cholesterol Education Program Adult Treatment Panel III (2001) (NCEP/ATP-III) and criteria for clinical diagnosis of metabolic syndrome in Iranian adults (CCDMIA).

\section{RESULTS}

Among the refereed individuals, 2980 peoples were aged $\geq 18$ years with male to women ratio of $1: 2.45$. NAFLD was diagnosed by ultrasound in $32.9 \%$ and $27.4 \%$ of men and women, respectively. MetS was detected in 65.9 and 64.6 of the patients with NAFLD (based on NCEP/ATP-III) and in $30.1 \%$ and $73.7 \%$ (based on CCDMIA) of men and women, respectively. There were no significant differences between two gender in none of the components $(p>0.05)$. Although, OR for hyperglycemia and abdominal obesity were approximately high in CCDMIA criteria (0.9613 and 1.2082, respectively), the differences were not statistically significant.

\section{CONCLUSION}

NAFLD was associated with MetS. However, it was not possible to determine whether NAFLD predating the development of MetS.

\section{KEYWORDS}

Non-alcoholic fatty liver disease, Metabolic syndrome, NCEP/ATP-III, Waist circumference, Hyperglycemia

Please cite this paper as:

Fattahi MR, Niknam R, Safarpour AR, Sepehrimanesh M, Lotfi M. The Prevalence of Metabolic Syndrome In Non-alcoholic Fatty Liver Disease; A Population-Based Study. Middle East J Dig Dis 2016;8:131-137. DOI: 10.15171/mejdd.2016.18

\section{INTRODUCTION}

Non-alcoholic fatty liver disease (NAFLD) comprises a disease spectrum ranging from simple steatosis to steatohepatitis, with different degrees of inflammation and fibrosis, which can progress to end-stage liver disease with cirrhosis and hepatocellular carcinoma. ${ }^{1}$ NAFLD is now more common than 
alcoholic liver disease owing to the rapid rise in the prevalence of obesity, and NAFLD is the most common cause of abnormal liver function tests. ${ }^{2}$ Previous studies reported that approximately $80 \%$ of cases with elevated liver enzyme levels in the American ${ }^{3}$, Japanese ${ }^{4}$, and Ital$\operatorname{ian}^{5}$ populations actually have NAFLD. Also, it has been demonstrated that NAFLD and NASH are common and may lead to serious clinical consequences. ${ }^{6}$ One possible risk factor of NAFLD is metabolic syndrome (MetS), which is becoming increasingly common. ${ }^{7,8}$ NAFLD affects $30 \%$ of the general adult population and up to 60 $70 \%$ of obese patients and those with diabetes mellitus. ${ }^{9}$

MetS is the term given to a cluster of dangerous risk factors for cardiovascular disease. MetS includes diabetes mellitus with raised fasting plasma glucose, abdominal obesity, hypertension, and hyperlipidemia. ${ }^{10,11}$ Some epidemiological evidence has shown the possible relationship between NAFLD and MetS., ${ }^{3,12}$ It has been reported that $30.1 \%$ of the Iranian population have $\mathrm{MetS}^{13}$, but there are no reports on the prevalence of this syndrome among individuals with NAFLD. NAFLD and MetS have been linked by relationships between central obesity, steatosis, and insulin resistance. ${ }^{8}$ Growing experimental and epidemiological evidence suggests that NAFLD and MetS share common interactions, but the definite evidence of a link between NAFLD and MetS is uncertain due to the small study populations. ${ }^{14}$

We therefore analyzed the prevalence of NAFLD and MetS in a large population registered to Kavar Cohor Study Center with respect to the two following research questions: First, what is the prevalence of MetS in NAFLD? Second, what is the role of each component of MetS in NAFLD existence? We also compared the diagnostic criteria of MetS based on the National Cholesterol Education Program Adult Treatment Panel III (NCEP/ ATP-III $)^{15}$ and criteria for clinical diagnosis of MetS in Iranian adults (CCDMIA). ${ }^{16}$

\section{MATERIALS AND METHODS}

\section{Study Population}

The present study is part of the Kavar Cohort Study (K.C.S) which started from 2006 in Kavar town with a population of about 71856. This town is located 35 kilometer southeast of Shiraz, the capital of Fars prov- ince, Iran. The Gastroenterohepatology Research Center (GEHRC) and Endocrine Research Center affiliated to Shiraz University of Medical Sciences are implementing K.C.S. From the start of K.C.S until now, all people without any age or sex exclusion criteria are followed every two years. The lifestyle nature of this town is rural and in the original cohort study, demographic and anthropometric characteristics of the participants were documented in the questionnaire. The questioners were trained on how to fill the questionnaire and how to measure personal characteristics such as waist circumference (WC), hip circumference (HC), height, weight, and systolic and diastolic blood pressures in a 3 month training program. All laboratory parameters were checked under international standards in the specialized laboratory of GEHRC. The blood specimens were also transferred to this lab in less than an hour under standard conditions. All the participants received oral information concerning the study and gave their written consent. The study was approved by the Ethics Committee of Shiraz University of Medical Sciences. We included $\geq 18$-year-old volunteers who had undergone ultrasonography confirming fatty liver. We excluded those who were alcohol users, active illicit drug abusers, were being treated for NAFLD or using medications causing fatty liver disease (such as amiodarone, tamoxifen and etc.), or were pregnant or seropositive for $\mathrm{HBC}, \mathrm{HCV}$, and HIV, or had any known liver disease.

\section{Measurements}

In all subjects, the diagnosis of NAFLD was made with demonstration of areas of hyperechogenicity on sonography. Sonograms were obtained with commercially available units using static and real-time scanning. A 3.5$\mathrm{MHz}$ transducer was routinely used, with a $2.25-\mathrm{MHz}$ transducer reserved for areas difficult to penetrate. The patients were examined in both supine and left posterior oblique positions, with longitudinal, transverse, and oblique scanning planes. The severity of echogenicity was graded as follows: grade 0, normal echogenicity; grade 1, slight, diffuse increase in fine echoes in liver parenchyma with normal visualization of diaphragm and intrahepatic vessel borders; grade 2, moderate, diffuse increase in fine echoes with slightly impaired visualization of intrahepatic vessels and diaphragm; grade 3, marked increase in fine echoes with poor or non-visu- 
Table 1: Two Criteria for clinical diagnosis of metabolic syndrome which was used in this study

\begin{tabular}{lcc}
\hline & \multicolumn{2}{c}{ Criteria } \\
\hline Variables & NCEP/ATP-III & CCDMIA \\
\hline Abdominal obesity & WC $>102 \mathrm{~cm}$ in men and $>88 \mathrm{~cm}$ in women & WC $\geq 95$ in both sexes \\
\hline Hypertriglyceridemia & $\mathrm{TG} \geq 150 \mathrm{mg} / \mathrm{dL}$ & $\mathrm{TG} \geq 150 \mathrm{mg} / \mathrm{dL}$ \\
\hline Low HDL-C level & $<40 \mathrm{mg} / \mathrm{dL}$ in men and $<50 \mathrm{mg} / \mathrm{dL}$ in women & $<40 \mathrm{mg} / \mathrm{dL}$ in men and $<50 \mathrm{mg} / \mathrm{dL}$ in women \\
\hline Hypertension & $\mathrm{SBP} / \mathrm{DBP} \geq 130 / 85 \mathrm{mmHg}$ & $\mathrm{SBP} / \mathrm{DBP} \geq 130 / 85 \mathrm{mmHg}$ \\
\hline Hyperglycemia & $\mathrm{FBS} \geq 110 \mathrm{mg} / \mathrm{dL}$ & $\mathrm{FBS} \geq 100 \mathrm{mg} / \mathrm{dL}$ \\
\hline $\begin{array}{l}\text { NCEP/ATP-III, National Cholesterol Education Program Adult Treatment Panel III; CCDMIA, Criteria for Clinical Diagnosis of metabolic syndrome in Iranian Adults; WC, Waist } \\
\text { Circumference; TG, Triglyceride, SBP, Systolic Blood Pressure; DBP, Diastolic Blood Pressure; FBS, Fasting Blood Sugar. }\end{array}$
\end{tabular}

Table 2: Frequency, percentage and mean \pm SD of the age of the participants based on different categories

\begin{tabular}{|c|c|c|c|c|c|}
\hline \multirow{2}{*}{ Variables } & \multirow{2}{*}{ Grade $^{\mathrm{a}}$} & \multicolumn{2}{|c|}{ Men } & \multicolumn{2}{|c|}{ Women } \\
\hline & & Frequency (\%) & Age (years) & Frequency (\%) & Age (years) \\
\hline$\geq 18$ years old & & $864(29)$ & $44.1 \pm 16.4 *$ & $2116(71)$ & $40.2 \pm 30.7$ \\
\hline \multirow{4}{*}{ NAFLD } & All & 285 (32.9) & $46.5 \pm 13.4$ & 579 (27.4) & $46.5 \pm 12.0$ \\
\hline & I & $180(63.2)$ & $45.5 \pm 13.6$ & $355(61.3)$ & $44.6 \pm 11.2$ \\
\hline & II & $88(30.9)$ & $47.9 \pm 12.2$ & $184(31.8)$ & $49.2 \pm 12.3$ \\
\hline & III & $17(5.9)$ & $50.4 \pm 15.9$ & $40(6.9)$ & $50.2 \pm 13.9$ \\
\hline
\end{tabular}

a In non-alcoholic fatly liver disease (NAFLD) in sonography: Grade I, mild; II, moderate; III, severe. Significant difference in age between men and women is indicated by asterisk

alization of the intrahepatic vessel borders, diaphragm, and posterior right lobe of the liver. ${ }^{17,18}$

WC between the lowest rib and the iliac crest at the level of umbilicus were measured in duplicate to the $\mathrm{cm}$ with flexible tape. Blood pressure was measured in sitting position with a random zero sphygmomanometer. Systolic and diastolic blood pressure was measured as duplicate on the left upper arm and the average used for analysis. Plasma glucose was measured by the glucoseperoxidase colorimetric enzymatic method with a sensitivity of $5 \mathrm{mg} / \mathrm{dL}$ and intra-assay coefficients of variation (CV) I.7\% in lower limit and $1.4 \%$ in upper limit concentrations. Inter-assay $\mathrm{CV}$ for the assay was $1.1 \%$ in lower limit and $0.6 \%$ in upper limit concentrations. Serum cholesterol and triglyceride of all the participants were measured after 12-14 hours of fasting with colorimetric method with a sensitivity of $5 \mathrm{mg} / \mathrm{dl}$. Intra-assay and inter-assay $\mathrm{CV}$ for the assay was $1.6 \%$ and $1.1 \%$ in lower limit and $0.6 \%$ and $0.9 \%$ for upper limit concentrations, respectively. High-density lipoprotein cholesterol (HDL-C) was measured after precipitation of the apolipoprotein B containing lipoproteins with phosphotungstic acid (Pars azmoon kit, Iran).

\section{Metabolic Syndrome criteria}

We used two different criteria, NCEP/ATP-III and CCDMIA. MetS was defined as the presence of three or more of the presented parameters in table $1 .^{15,16}$

\section{Statistical Analyses}

This study was a descriptive study and the data were collected and stored in a computer database. Missing values and data entry errors were checked and related subjects were deleted, if data correction was not possible. Then, statistical analyses was performed using descriptive analysis and also Chi-square test using SPSS software, version 16 (SPSS Ltd., Woking, Surrey, UK) for Windows at $p<0.05$. The data were presented as frequencies, percentages and $95 \%$ confidence intervals.

\section{RESULTS}

We enrolled 1069 (31.3\%) men and 2346 (68.7\%) women. Among the individuals $\geq 18$ years there were 864 $(80.8 \%)$ men and $2116(90.2 \%)$ women with a male to female ratio of $1: 2.45$. Table 2 shows the frequency, percentage and mean \pm SD age of all participants, those $\geq 18$ years, and those $\geq 18$ years that had NAFLD with different 
Table 3: Frequency (\%) of metabolic syndrome (MetS) in different sonographic gradings of non-alcoholic fatty liver disease (NAFLD)

\begin{tabular}{cccc}
\hline \multirow{2}{*}{ MetS criteria } & \multirow{2}{*}{ NAFLD grade } & \multicolumn{2}{c}{ Frequency (\%) } \\
\cline { 2 - 4 } & & Men & Women \\
\hline \multirow{2}{*}{ NCEP/ATPIII } & & $188(65.9)$ & $376(64.6)$ \\
\cline { 2 - 4 } & I & $97(51.6)$ & $189(50.4)$ \\
\cline { 2 - 4 } & II & $65(34.6)$ & $148(39.4)$ \\
\hline \multirow{2}{*}{ CCDMII } & $26(13.8)$ & $39(10.2)$ \\
\cline { 2 - 4 } & & $103(30.1)$ & $429(73.7)$ \\
\cline { 2 - 4 } & I & $53(51.7)$ & $214(50.0)$ \\
\cline { 2 - 4 } & II & $35(33.8)$ & $161(37.4)$ \\
\hline
\end{tabular}

NCEP/ATP-III, National Cholesterol Education Program Adult Treatment Panel III; CCDMIA, Criteria for Clinical Diagnosis of MetS in Iranian Adults.

Table 4: Prevalence of the components of metabolic syndrome (MetS) in patients with NAFLD based on different criteria and sex

\begin{tabular}{|c|c|c|c|c|c|}
\hline \multirow{2}{*}{ MetS components } & & \multicolumn{2}{|c|}{ Frequency (percentage) } & \multirow{2}{*}{$95 \% \mathrm{CI}$} & \multirow{2}{*}{ Odds ratio } \\
\hline & & Men & Women & & \\
\hline \multirow{2}{*}{ Abdominal obesity (NCEP/ATPIII) } & Yes & $56(19.6)$ & $436(74.9)$ & \multirow{2}{*}{$0.0579-0.1159$} & \multirow{2}{*}{0.0819} \\
\hline & No & $229(80.4)$ & $146(25.1)$ & & \\
\hline \multirow{2}{*}{ Abdominal obesity (CCDMIA) } & Yes & $149(52.3)$ & $310(53.3)$ & \multirow{2}{*}{$0.7238-1.2768$} & \multirow{2}{*}{0.9613} \\
\hline & No & $136(47.7)$ & $272(46.7)$ & & \\
\hline \multirow{2}{*}{ Hyperglycemia (NCEP/ATPIII) } & Yes & $56(19.6)$ & $123(21.1)$ & \multirow{2}{*}{$0.6408-1.2995$} & \multirow{2}{*}{0.9126} \\
\hline & No & $229(80.4)$ & $459(78.9)$ & & \\
\hline \multirow{2}{*}{ Hyperglycemia (CCDMIA) } & Yes & $113(39.6)$ & $205(35.2)$ & \multirow{2}{*}{$0.9022-1.6179$} & \multirow{2}{*}{1.2082} \\
\hline & No & $172(60.4)$ & $377(64.8)$ & & \\
\hline \multirow{2}{*}{ Hypertriglyceridemia } & Yes & $135(50.9)$ & $435(74.7)$ & \multirow{2}{*}{$0.2587-0.476$} & \multirow{2}{*}{0.3509} \\
\hline & No & $130(49.1)$ & $147(25.3)$ & & \\
\hline \multirow{2}{*}{ Low HDL level } & Yes & $115(43.7)$ & $320(55.0)$ & \multirow{2}{*}{$0.4745-0.853$} & \multirow{2}{*}{0.6362} \\
\hline & No & $148(56.3)$ & $262(45.0)$ & & \\
\hline \multirow{2}{*}{ Hypertension } & Yes & $103(36.1)$ & $231(39.7)$ & \multirow{2}{*}{$0.6414-1.1529$} & \multirow{2}{*}{0.8599} \\
\hline & No & $182(63.9)$ & $351(60.3)$ & & \\
\hline
\end{tabular}

NCEP/ATP-III, National Cholesterol Education Program Adult Treatment Panel III; CCDMIA, Criteria for Clinical Diagnosis of MetS in Iranian Adults.

grades. The men to women ratio in all participants, those $\geq 18$ years, and those $\geq 18$ years who had NAFLD were $1: 2.19,1: 2.45$, and 1:2.03, respectively. Mild NAFLD was more prevalent in both men and women. However, moderate and severe NAFLD was more prevalent in women compared with men $(p>0.05)$. Among those $\geq 18$ years, men were about 4 years older than women $(p<0.001)$. NAFLD was detected in $33.1 \%$ and $27.4 \%$ of men and women, respectively.

The prevalence of MetS and its related NAFLD grades according to NCEP/ATPIII and CCDMIA are presented in table 3. As demonstrated, the number of men with MetS based on NCEP/ATPIII was higher than CCDMIA. In the opposite trend, the number of women with MetS based on CCDMIA was higher than NCEP/ATPII. Among men, according to the NCEP/ATPIII and CCDMIA criteria, $65.9 \%$ and $30.1 \%$ had MetS, respectively. These percentages in women were $64.6 \%$ and $73.7 \%$, based on NCEP/ATPIII and CCDMIA criteria, respectively. Also, most of the patients with MetS had mild NAFLD in both sexes based on both criteria (table 2).

The frequency, percentage, $95 \% \mathrm{CI}$, and odds ratio for each component of MetS in patients with NAFLD is presented in table 4 . This demonstrated that there were no sig- 
nificant differences between the two sexes in none of the components $(p>0.05)$. Although, OR for hyperglycemia and abdominal obesity were approximately high in CCDMIA criteria (0.9613 and 1.2082, respectively), the respected differences were not statistically significant.

\section{DISCUSSION}

This study assessed the prevalence of MetS and its associated components in different grades of NAFLD in called and refereed volunteers from the K.C.S as the first study from the Middle East. We found no significant difference between men and women in NAFLD prevalence nor in MetS based on both criteria. There is disagreement between researchers about the methods of NAFLD diagnosis. The diagnosis of NAFLD requires a combination of invasive and non-invasive tests. Mild to moderately increased serum levels of aspartate aminotransferase and alanine aminotransferase or both were the most common findings. ${ }^{19}$ However some studies suggest that the use of liver enzymes as a marker of NAFLD underestimate its prevalence. ${ }^{20}$ Ultrasound has a sensitivity of $89 \%$ and $77 \%$ and a specificity of $93 \%$ and $89 \%$ in detecting steatosis and increased fibrosis, respectively, ${ }^{19}$ and therefore we used this noninvasive easy technique in our study.

The prevalence of NAFLD was determined to be $25.7 \%$ in this study, which is different from the one report from Taiwan. ${ }^{21}$ Other reports show rates of $10-30 \%$ from Taiwan $^{22}$, Japan ${ }^{4}$, India ${ }^{23}$, and USA, Europe, and Indonesia. ${ }^{24,25}$ Also, the prevalence of MetS in patients with NAFLD ranged from 61-65\%, based on CCDMIA and NCEP/ATPIII, respectively which was 1.8 to 3.1 times more than previous reports. ${ }^{10}$ This is moderately higher than that reported by Uchil et al. among Indian adult patients with NAFLD (47\%). ${ }^{19}$ The higher prevalence of NAFLD in men compared with women in our study (33.1\% vs. $27.5 \%$ ) was similar to a previous report from Taiwan. ${ }^{19}$ Despite the difference in criteria for the two components of MetS and sex, the prevalence of mild NAFLD (grade I) in patients with MetS was approximately high (about 50\%). In the present study, hyperglycemia (OR: 1.21, 95\% CI: 0.90-1.62) and abdominal obesity (OR: 0.96, 95\% CI: 0.72-1.28), based on CCDMIA were the most NAFLD related components of MetS. NAFLD and MetS have been tangentially linked in the association with central obesity and insulin resistance. ${ }^{8}$ This fact was highlighted in a large multicenter study, which showed that metabolic syndrome and insulin resistance were associated with NAFLD by multivariate analysis. ${ }^{26}$ It has been reported that the prevalence of MetS in the Iran is one of the highest worldwide. In the adult population of the Tehran Lipid and Glucose Study (TLGS), MetS was found in $42 \%$ of women and $24 \%$ of men with a total age-standardized prevalence of $33.7 \% .{ }^{27}$ Also, it has been reported that obesity is now recognized as a major health problem and is the prominent underlying factor in MetS. ${ }^{10}$ The findings from our study support the relationship between obesity and hyperglycemia with MetS. In a report from Australia, patients with NAFLD were more likely to develop MetS on follow-up. ${ }^{27}$ Marchesani et al. showed that $80 \%$ of patients with NAFLD were obese ${ }^{29}$ that is obviously higher than our estimation $(47.2 \%$ in NCEP/ATPIII and 52.8\% in CCDMIA).

The prevalence of MetS differed widely in different studies according to the population sample studied and the diagnostic criteria used. In our study population, the prevalence of MetS (65.25\% and 51.90\%) was different based on the diagnostic criteria used (NCEP/ATP-III or CCDMIA). The prevalence of MetS tended to be higher in men when the NCEP/ATP-III criteria were used, and tended to be higher in women when the CCDMIA were used. For these two definitions, the greatest difference is of the diagnosis of hyperglycemia and abdominal obesity.

Our study suffered from three important limitations: First, in our participants, volunteers women were more refereed than men due to occupational busy of men; Second, NAFLD diagnosis and grading was made only based on sonography. The most important reasons for this limitation were performing sonography in asymptomatic volunteer population and also its inexpensiveness and non-invasiveness; Third, our participants did not have any control group.

In conclusion, although our study demonstrated that NAFLD was associated with MetS, from this analysis it was not possible to determine whether NAFLD predating the development of MetS. Moreover, difference in detected MetS prevalence between NCEP/ATP-III and CCDMIA demonstrated that criteria in MetS are still a controversial subject and definition of better and more 
effective criteria for diagnosis of MetS in different population is seriously needed. Further well-designed case-control studies with follow-up and using of concurrent different criteria are needed to elucidate the causative relationship between these two conditions.

\section{ACKNOWLEDGMENT}

This study was conducted as a part of Kavar Cohort Study. The authors wish to thank all staff of Gastroeneterohepatology Research Center, Shiraz University of Medical Sciences, Shiraz, Iran for their kindly help in performing of this study.

\section{CONFLICT OF INTEREST}

The authors declare no conflict of interest related to this work.

\section{REFERENCES}

1. Pacifico L, Nobili V, Anania C, Verdecchia P, Chiesa C. Pediatric nonalcoholic fatty liver disease, metabolic syndrome and cardiovascular risk. World $J$ Gastroenterol 2011;17:3082-91.

2. Sattar N, Forrest E, Preiss D. Non-alcoholic fatty liver disease. Brit Med J 2014;349:g4596. doi: 10.1136/bmj.g4596.

3. Marchesini G, Bugianesi E, Forlani G, Cerrelli F, Lenzi M, Manini R, et al. Nonalcoholic fatty liver, steatohepatitis, and the metabolic syndrome. Hepatology 2003;37:91723. doi: $10.1053 /$ jhep. 2003.50161

4. Nomura H, Kashiwagi S, Hayashi J, Kajiyama W, Tani S, Goto M. Prevalence of fatty liver in a general population of Okinawa, Japan. Jpn J Med 1988;27:142-9. doi:10.2169/internalmedicine1962.27.142

5. Bellentani S, Saccoccio G, Masutti F, Croce LS, Brandi $\mathrm{G}$, Sasso F, et al. Prevalence of and risk factors for hepatic steatosis in Northern Italy. Ann Intern Med 2000;132:1127. doi:10.7326/0003-4819-132-2-200001180-00004

6. Sotoudehmanesh R, Sotoudeh M, Ali-Asgari A, AbediArdakani B, Tavangar SM, Khakinejad A, et al. Silent liver diseases in autopsies from forensic medicine of Tehran. Arch Iran Med 2006;9:324-8.

7. Musso G, Gambino R, Tabibian JH, Ekstedt M, Kechagias S, Hamaguchi M, et al. Association of non-alcoholic fatty liver disease with chronic kidney disease: a systematic review and meta-analysis. PLoS Med 2014;11:e1001680. doi: 10.1371/journal.pmed.1001680

8. Streba LAM, Carstea D, Mitruţ P, Vere CC, Dragomir N, Streba CT. Nonalcoholic fatty liver disease and metabolic syndrome: a concise review. Rom J Morphol Embryol 2008;49:13-20.

9. Chalasani N, Younossi Z, Lavine JE, Diehl AM, Brunt
EM, Cusi K, et al. The diagnosis and management of non-alcoholic fatty liver disease: Practice Guideline by the American Association for the Study of Liver Diseases, American College of Gastroenterology, and the American Gastroenterological Association. Hepatology 2012;55:2005-23. doi: 10.1002/hep.25762

10. Chen SH, He F, Zhou HL, Wu HR, Xia C, Li YM. Relationship between nonalcoholic fatty liver disease and metabolic syndrome. J Digest Dis 2011;12:125-30. doi: 10.1111/j.1751-2980.2011.00487.x

11. Cho LW. Metabolic syndrome. Singapore Med J 2011;52:779-85

12. Tsai C-H, Li T-C, Lin C-C. Metabolic syndrome as a risk factor for nonalcoholic fatty liver disease. South Med $J$ 2008;101:900-5. doi: 10.1097/SMJ.0b013e31817e8af9

13. Hadaegh F, Zabetian A, Harati H, Azizi F. Metabolic syndrome in normal-weight Iranian adults. Ann Saudi Med 2007;27:18-24.

14. Sharifi F, Mousavinasab SN, Saeini M, Dinmohammadi M. Prevalence of metabolic syndrome in an adult urban population of the west of Iran. Exp Diabetes Res 2009;2009:136501-5. doI: 10.1155/2009/136501

15. National Heart Lung and Blood Institute. Third report of the National Cholesterol Education Program (NCEP) expert panel on detection, evaluation, and treatment of high blood cholesterol in adults (Adult Treatment Panel III): final report. Circulation 2002;106:3143-421. doi:10.1001/ jama.285.19.2486

16. Azizi F, Hadaegh F, Khalili D, Esteghamati AR, Hosseinpanah F, Delavari AR, et al. Appropriate definition of metabolic syndrome among Iranian adults: Report of the Iranian National Committee of Obesity. Arch Iran Med 2010;13:426-8.

17. Quinn SF, Gosink BB. Characteristic sonographic signs of hepatic fatty infiltration. Am J Roentgenol 1985;145:753-5.

18. Saadeh S, Younossi ZM, Remer EM, Gramlich T, Ong JP, Hurley M, et al. The utility of radiological imaging in nonalcoholic fatty liver disease. Gastroenterology 2002;123:745-50. doi: 10.1053/gast.2002.35354

19. Uchil D, Pipalia D, Chawla M, Patel R, Maniar S, Narayani, et al. Non-alcoholic fatty liver disease (NAFLD)-the hepatic component of metabolic syndrome. J Assoc Physicians India 2009;57:201-4.

20. Zelber-Sagi S, Nitzan-Kaluski D, Halpern Z, Oren R. Prevalence of primary non-alcoholic fatty liver disease in a population-based study and its association with biochemical and anthropometric measures. Liver Int 2006;26:856-63. doi: 10.1111/j.1478-3231.2006.01311.x

21. Chen C-H, Huang M-H, Yang J-C, Nien C-K, Yang C-C, Yeh Y-H, et al. Prevalence and risk factors of nonalcoholic fatty liver disease in an adult population of Taiwan: metabolic significance of nonalcoholic fatty liver disease in nonobese adults. J Clin Gastroenterol 2006;40:745-52.

22. Hsiao PJ, Kuo KK, Shin SJ, Yang YH, Lin WY, Yang 
JF, et al. Significant correlations between severe fatty liver and risk factors for metabolic syndrome. $J$ Gastroenterol Hepatol 2007;22:2118-23. doi:10.1111/j.14401746.2006.04698.x

23. Singh SP, Nayak S, Swarn M. Prevalence of NAFLD in eastern coastal India-A Preliminary USG study. Trop Gastroenterol 2004;25:76-9.

24. Chitturi S, Farrell GC, George J. Non-alcoholic steatohepatitis in the Asia-Pacific region: Future shock? J Gastroenterol Hepatol 2004;19:368-74. doi: 10.1111/j.14401746.2003.03252.x

25. Farrell GC. Non-alcoholic steatohepatitis: What is it, and why is it important in the Asia-Pacific region? J Gastroenterol Hepatol 2003;18:124-38. doi: 10.1046/j.1440-1746. 2003.02989.x

26. Caballería L, Pera G, Auladell MA, Torán P, Muñoz L, Miranda D, et al. Prevalence and factors associated with the presence of nonalcoholic fatty liver disease in an adult population in Spain. Eur J Gastroenterol Hepatol 2010;22:24-32. doi: 10.1097/MEG.0b013e32832fcdf0

27. Azizi F, Salehi P, Etemadi A, Zahedi-Asl S. Prevalence of metabolic syndrome in an urban population: Tehran Lipid and Glucose Study. Diabetes Res Clin Pract 2003;61:2937. doi: 10.1016/S0168-8227(03)00066-4

28. Adams LA, Waters OR, Knuiman MW, Elliott RR, Olynyk JK. NAFLD as a risk factor for the development of diabetes and the metabolic syndrome: an eleven-year follow-up study. Am J Gastroenterol 2009;104:861-7. doi:10.1038/ajg.2009.67

29. Marchesini G, Brizi M, Bianchi G, Tomassetti S, Bugianesi E, Lenzi M, et al. Nonalcoholic fatty liver disease a feature of the metabolic syndrome. Diabetes 2001;50:1844-50. doi: $10.2337 /$ diabetes.50.8.1844 